\title{
Estimación de la concentración de clorofila mediante métodos no destructivos en vid (Vitis vinifera L.) cv. Riesling Becker
}

\section{Chlorophyll concentration estimation using non-destructive methods in grapes (Vitis vinifera L.) cv. Riesling Becker}

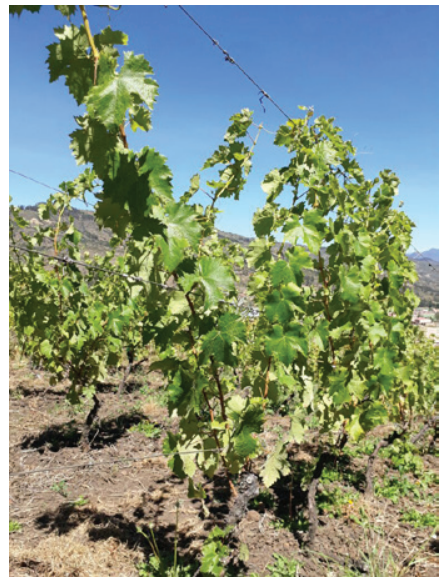

CRISTIAN SANTIAGO CASTAÑEDA ${ }^{1,4}$

PEDRO JOSÉ ALMANZA-MERCHÁN²

ELBERTH HERNANDO PINZÓN ${ }^{3}$

GERMÁN EDUARDO CELY-REYES²

PABLO ANTONIO SERRANO-CELY2

Cultivo de Vitis vinifera, cv. Riesling Becker.

Foto: C.S. Castañeda

\section{RESUMEN}

El análisis de pigmentos en plantas es un procedimiento de laboratorio y comúnmente no es una medida inmediata que se realice in situ. El SPAD 502 y CCM-200 son equipos para estimar de modo indirecto, no destructivo y rápido el contenido de clorofila. La concentración de pigmentos fotosintéticos se relaciona con la concentración foliar de nitrógeno, por lo que de forma indirecta se puede conocer la deficiencia o exceso del elemento, puede servir como fundamento técnico para sugerir el manejo adecuado del cultivo, en busca de potencializar la eficiencia fotosintética, calidad y el rendimiento de un viñedo. La investigación buscó comparar las mediciones entre el medidor portátil de clorofila SPAD-502 y CCM-200, en la variedad de uva Riesling Becker, bajo condiciones de clima frío tropical, y con ello determinar el comportamiento de clorofila durante el crecimiento de las hojas 4, 5 y 6, desde floración hasta vendimia. La relación entre las mediciones con el SPAD502 y el CCM-200 se ajustaron a un polinomio de segundo grado determinado por la ecuación, para el Índice de Concentración de Clorofila, como ICC $=0,014$ SPAD $^{2}-0,2396 \mathrm{SPAD}+5,8021$. Con un valor de $R^{2}=0,9343$ $(n=96 ; P \leq 0,0001)$, el cual indica que existe una alta correlación entre los equipos portátiles empleados en el presente estudio para determinar la clorofila de forma no destructiva. Las evaluaciones realizadas con los equipos permitieron obtener medidas rápidas y confiables. Siendo importante la elección del momento de

\footnotetext{
Facultad de Ciencias Agropecuarias, Programa de Maestría en Fisiología Vegetal, Grupo de Investigación GIPSO, Universidad Pedagógica y Tecnológica de Colombia (UPTC), Tunja (Colombia). ORCID Castañeda, C.S.: 0000-0002-17725628

2 Facultad de Ciencias Agropecuarias, Grupo de Investigaciones GIPSO, Universidad Pedagógica y Tecnológica de Colombia (UPTC), Tunja (Colombia). ORCID Almanza-Merchán, P.J.: 0000-0002-9207-0617; ORCID Cely-Reyes, G.E.: 0000-0001-6312-3575; ORCID Serrano-Cely, P.A.: 0000-0002-1270-3024

3 Facultad de Ciencias Agropecuarias, Grupo de Investigaciones Agrícolas, Universidad Pedagógica y Tecnológica de Colombia (UPTC), Tunja (Colombia). ORCID Pinzón, E.H: 0000-0001-9229-3450

4 Autor para correspondencia. cscaser30@hotmail.com
} 
medición de acuerdo con el estadio fenológico de la planta debido a la alta variabilidad en los contenidos de clorofila que se presentan en relación a la filotaxia.

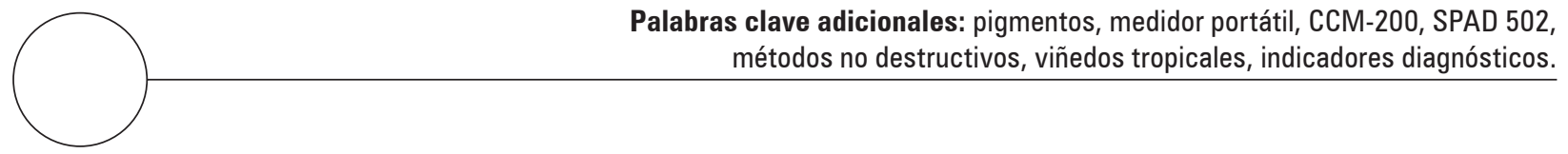

\section{ABSTRACT}

Pigment analysis is a laboratory procedure and is not an immediate measurement to be performed in situ. The SPAD 502 and CCM-200 are equipments used for non-destructive, fast, and indirect estimates of chlorophyll contents. The concentration of photosynthetic pigments is related to the foliar concentration of nitrogen, so indirectly, it is possible to observe the deficiency or excess of this element, providing a technical basis to suggest the proper management of the crop in order to potentiate the photosynthetic efficiency, quality and yield of a vineyard. This research sought to compare measurements between the portable chlorophyll meter SPAD-502 vs. CCM-200 in the Riesling Becker grape variety under cold tropical climate conditions to determine the behavior of chlorophyll during the growth of leaves 4,5 and 6 , from flowering to harvest. The relationship between the measurements with the SPAD-502 and the CCM-200 were fit to a second degree polynomial determined with the equation for the Chlorophyll Concentration Index: CCI $=0.014 \mathrm{SPAD} 2-0.2396 \mathrm{SPAD}+5.8021$. With a value of $R^{2}=0.9343(n=96$; $P \leq 0.0001$ ), which indicates that there was a high correlation between the portable equipment used in the present study to determine chlorophyll with a non-destructive method. The evaluations carried out with the equipment obtained fast and reliable measurements, with the choice of the moment of measurement according to the phenological stage of the plant being important because of the high variability in chlorophyll contents that are presented in relation to the phylotaxy.

Additional key words: pigments, portable meter, CCM-200, SPAD 502, non-destructive methods, tropical vineyards, diagnostic indicators.

Fecha de recepción: 28-11-2017 Aprobado para publicación: 30-05-2018

INTRODUCCIÓN

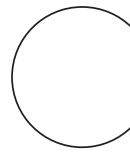

En Colombia se cuenta con pocas regiones que presentan ambientes favorables para el establecimiento del cultivo de vid destinada para la elaboración de vinos (Pinzón-Sandoval et al., 2017; Walteros et al., 2012). A pesar de ello, posee lugares adecuados por ser un país tropical con condiciones de radiación solar y temperaturas variables, que sumado a un adecuado manejo del cultivo pueden alcanzar hasta dos cosechas por año (Almanza-Merchán et al., 2012a). De acuerdo con Agronet (2018), en Colombia se registró una producción nacional de 30.000 t de uva para el año 2016, donde los departamentos de Valle del Cauca y Huila participaron con las mayores producciones, 24.379 y 5.286 t, respectivamente. La mayor producción de uva con fines vinícolas en el año 2016 se registró en Boyacá, con 106,5 t, siendo éste el departamento productor de uva para vino más importante en el país.
Quijano (2004) indicó que en este las condiciones climáticas, y en especial la luminosidad, se convierte en el factor fundamental para la obtención de uva para elaboración de vinos de alta calidad.

Almanza-Merchán et al. (2012b) señalan que gracias a las condiciones ambientales que presentan algunos municipios de las provincias del alto Ricaurte, Sugamuxi, Tundama, Valderrama y Norte en Boyacá, se pueden cultivar diversas variedades de uva con fines vitícolas. Quijano (2004) señala que desde hace 35 años se seleccionaron las variedades actuales que se adaptaron a las condiciones climáticas de la loma de Puntalarga, en donde se presentan condiciones equivalentes a la región de Alsacia en Francia, lugar de origen de algunas variedades como Riesling, Pinot Noir y Riesling Becker. Además, Almanza (2011) 
menciona como las regiones boyacenses en las cuales se encuentran situados los viñedos se caracterizan por poseer suelos de textura liviana, franco-arenosos y con fertilidad natural baja y que de acuerdo con el sistema de clasificación de zonas de vida propuestas por Holdrige, se presenta una sola zona de vida: bosque seco montano bajo (Bs-MB). Teniendo en cuenta las condiciones que poseen estas zonas productoras de uva se hace necesaria la implementación de nuevas tecnologías las cuales permitan un mejor control en el comportamiento fisiológico de las plantas, optimizando así el manejo del cultivo.

El contenido de clorofila en hojas se presenta como una de las variables importantes en el momento de evaluar el estatus fisiológico de las plantas y su relación entre las fuentes y vertederos (Do Amarante et al., 2009). Esto, facilitaría determinar en qué momento una planta se encuentra en circunstancias de estrés por salinidad o por cualquier otro tipo (Alizadeh et al., 2010; Delegido et al., 2010; Elarab et al., 2015), los cuales perjudican el proceso fotosintético y generan una reducción en la asimilación de nitrógeno y en la fijación del carbono, factores que modifican en la planta su concentración interna de clorofila alterando su coloración normal de la cual es responsable la molécula de clorofila (Steele et al., 2007).

Varios estudios indican la existencia de la relación directa entre el contenido de clorofila y el contenido de nitrógeno en hojas, debido a que este elemento es necesario dentro del proceso de síntesis de la molécula de clorofila, y así mismo se encuentra ligado en la fase luminosa del proceso fotosintético (Salisbury y Ross, 1992). Trabajos realizados por Sainz y Echeverria (1998), indican la importancia del uso de métodos indirectos como el clorofilómetro de tipo SPAD ${ }^{\circledR}$ (Konica Minolta, Osaka, Japón) como herramienta para el monitoreo de la disponibilidad de nitrógeno, asociada con el rendimiento del cultivo de maíz. De igual forma, Rincón y Ligarreto (2010) encontraron relación directa entre el contenido de nitrógeno y los valores obtenidos con el clorofilometro en plantas de maíz bajo diferentes dosis de nitrógeno. Ramírez et al. (2012) proponen el uso de medidores portátiles de clorofila como herramienta para la detección temprana de deficiencias de nitrógeno y disminuciones del rendimiento asociados a condiciones de estrés en plantas de café.

El uso de diferentes medidores portátiles de clorofila se fundamenta en que parte de la luz que llega a la hoja es absorbida por la clorofila y el resto que se refleja entra en contacto con la celda detectora de los equipos siendo está convertida en una señal eléctrica. De acuerdo con sus fundamentos de uso, los equipos presentan similitudes, pero difieren en cuanto al rango de longitud de onda utilizados. Es así como el CCM-200 ${ }^{\circledR}$ (Opti Sciences, Hudson, NH, USA) presenta longitudes de onda que van desde 665 (rojo) hasta $940 \mathrm{~nm}$ (infrarrojo) (Cate y Perkins, 2003; Cao et al., 2015). En tanto, el SPAD 502 (Konica Minolta, Osaka, Japón), utiliza longitudes de onda central de $650 \mathrm{~nm}$ (rojo) y $940 \mathrm{~nm}$ (infrarrojo) (Kapotis et al., 2003; Hawkins et al., 2009; Romano et al., 2011).

Po lo anterior, el presente trabajo tuvo como objetivo evaluar dos métodos no destructivos para estimar las concentraciones de clorofila en hojas de Vitis vinifera L. cv. Riesling Becker durante el crecimiento y desarrollo, en condiciones de clima frío tropical en el municipio de Corrales, Boyacá.

\section{MATERIALES Y MÉTODOS}

El estudio se desarrolló en el municipio de Corrales, departamento de Boyacá, situado a $5^{\circ} 46^{\prime} 47,1^{\prime \prime} \mathrm{N}$ y $72^{\circ} 58^{\prime} 36,5^{\prime \prime} \mathrm{W}$, a una altitud de $2.450 \mathrm{msnm}$. El clima de la zona se caracteriza por presentar una temperatura promedio anual de $16,5^{\circ} \mathrm{C}$ y precipitación bimodal media de $700 \mathrm{~mm}$ año ${ }^{-1}$. Se emplearon 10 plantas que presentaban una edad de 15 años y que correspondían a la selección clonal de Vitis vinifera L. cultivar Riesling $\times$ Silvaner, también conocida como Riesling Becker procedente de Alsacia, Alemania. Estás fueron sembradas a una distancia de $1,20 \times 0,90$ $\mathrm{m}$, el sistema de manejo es el de tipo guyot simple con conducción en espaldera a tres alambres.

Las plantas monitoreadas pertenecían a zonas del lote donde históricamente el cultivo demuestra buen crecimiento, desarrollo y frutos aptos para la elaboración de vinos. El plan de fertilización y el manejo fitosanitario se realizó según requerimiento del cultivo.

Cuando las plantas se encontraron en estadio principal 5 (inflorescencia claramente visible), de acuerdo con la escala de la BBCH propuesta para vid por Lorenz et al. (1994), fueron seleccionados tres pámpanos por planta a partir de los cuales según la filotaxia fueron marcadas las hojas 1 a 12 en cada uno. Con el fin de establecer el comportamiento de la concentración de clorofila en relación con la posición, se tomaron seis mediciones por hoja para posteriormente obtener un dato promedio, para evitar variación de los valores las mediciones fueron obtenidas de la parte basal media y apical de las hojas. La concentración de clorofila 
se determinó a través de los equipos portátiles para medición no destructiva CCM-200 (Opti Sciences, Tynsgboro, MA) y el SPAD 502 (Konica Minolta, Osaka, Japón). Las mediciones fueron realizadas cada $15 \mathrm{~d}$ con el fin de cubrir los estadios de crecimiento y desarrollo del fruto de vid hasta llegar al estadio principal 8 (maduración del fruto).

Los datos obtenidos fueron sometidos a pruebas de normalidad y homogeneidad mediante las pruebas Shapiro Wilk y Levene respectivamente, comprobando supuestos se procedió a realizar el análisis de varianza, sometiendo a pruebas de comparación de medias de Tukey $(P \leq 0,05)$. Para el análisis de los datos se empleó el programa estadístico $\mathrm{SAS}^{\circledR}$ v. 9.2 (Cary, NC, USA). Para la determinación de la correlación entre métodos se emplearon modelos de tipo polinomial.

\section{RESULTADOS Y DISCUSIÓN}

En relación con el contenido de clorofila evaluado a través de los dos métodos de medición de la hoja 1 a 12 hojas fueron encontradas diferencias significativas $(P \leq 0,05)$ para los dos métodos en relación a la posición de la hoja en función del racimo. El menor valor se encontró en la hoja 1 para los dos métodos evaluados con valores de 8,8 $\pm 0,28$ unidades de Índice de Concentración de Clorofila (ICC) y 33,8 $\pm 0,24$ unidades SPAD, mientras que las hojas 6 y 7 mostraron los valores más altos con $15,4 \pm 0,1$ y 15,1 $\pm 0,2$ unidades ICC respectivamente, y 43,9 $\pm 0,4$ y $44,4 \pm 0,7$ unidades SPAD respectivamente (Fig. 1).
Los resultados indican que los valores más altos para los dos métodos se presentan en el tercio medio de la planta (hojas 4 a 7). De acuerdo a la posición de la hoja, los resultados obtenidos son similares a los reportados por Novoa y Villagran (2002), quienes encontraron la mayor distribución de clorofila en el tercio medio en plantas de maíz. Así mismo, Ocon (2008) reporta que debido a la gran movilidad que posee el nitrógeno, las plantas logran acumular el mayor contenido de clorofila y $\mathrm{N}$ en las hojas ubicadas en su tercio medio. Numerosos estudios soportan como el comportamiento de la clorofila y el nitrógeno representan un valor proporcional al de las unidades SPAD y ICC en especies como Solanum lycopersicum cv. Río Grande (Rodríguez et al., 1998), Solanum tuberosum (Arregui et al., 2000; Borhan et al., 2017), Zea mays (Reyes et al., 2017) y Gossypium hirsutum (Rosolem y Mellis, 2010), especies para biodiesel como Jatropha curcas (Senger et al., 2014).

De igual manera, Zulini et al. (2007) reportan como en $V$. vinifera cv. 'White Riesling' se observa el mismo fenómeno donde a medida en que las hojas se acercan al ápice se marca la disminución de clorofila (medida como unidades SPAD) y aumentando en la parte media de los pámpanos. La cuantificación de la clorofila mediante los métodos CCM-200 y SPAD en relación al estadio fenológico presentó diferencias significativas según la prueba de Tukey $(P \leq 0,05)$, siendo el estadio de envero en el que se presentó los mayores valores con 14,1 $\pm 0,3$ unidades ICC y $43,4 \pm 0,2$ unidades SPAD, mientras que el estadio antesis se presentó valores de 7,2 $\pm 0,3$ unidades ICC y $33,2 \pm 0,9$ unidades SPAD siendo los menores valores (Fig. 2).
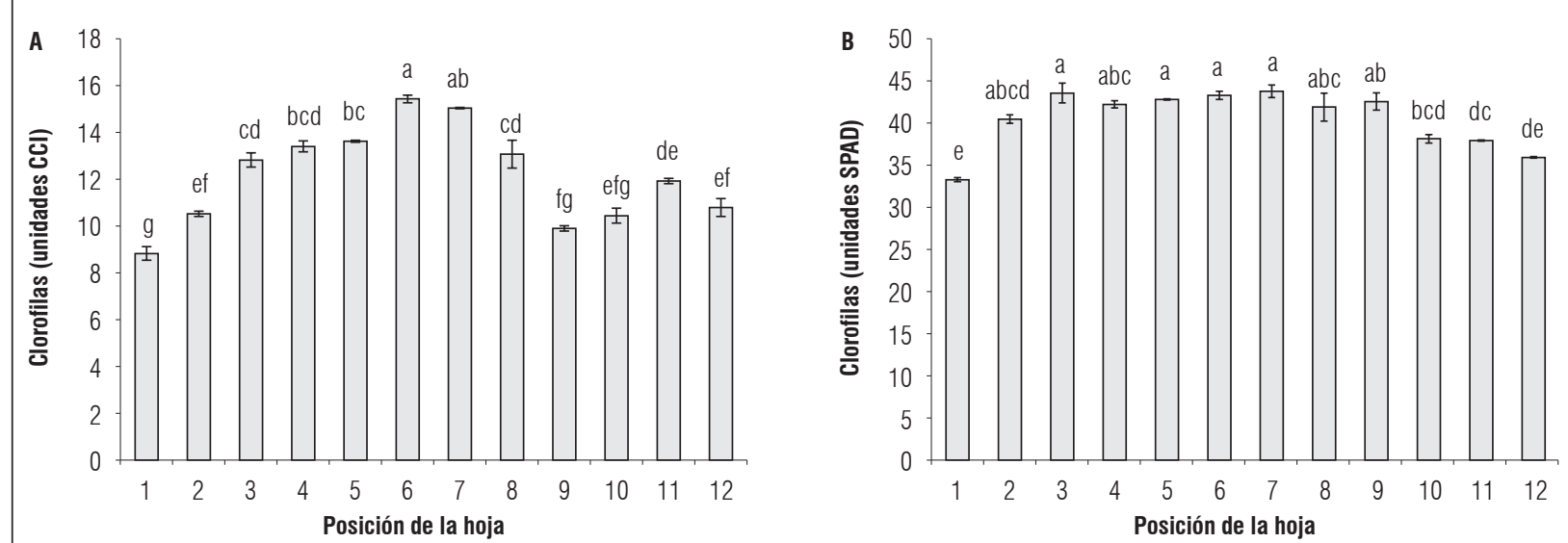

Figura 1. Contenido de clorofilas totales medido con: A. CCM-200 y B. SPAD 502 en hojas de Vitis vinifera L. cv. 'Riesling Becker' bajo condiciones de trópico alto. Promedios con letras distintas indican diferencia significativa según la prueba de Tukey $(P \leq 0,05)$. Barras verticales indican error estándar $(n=3)$. 

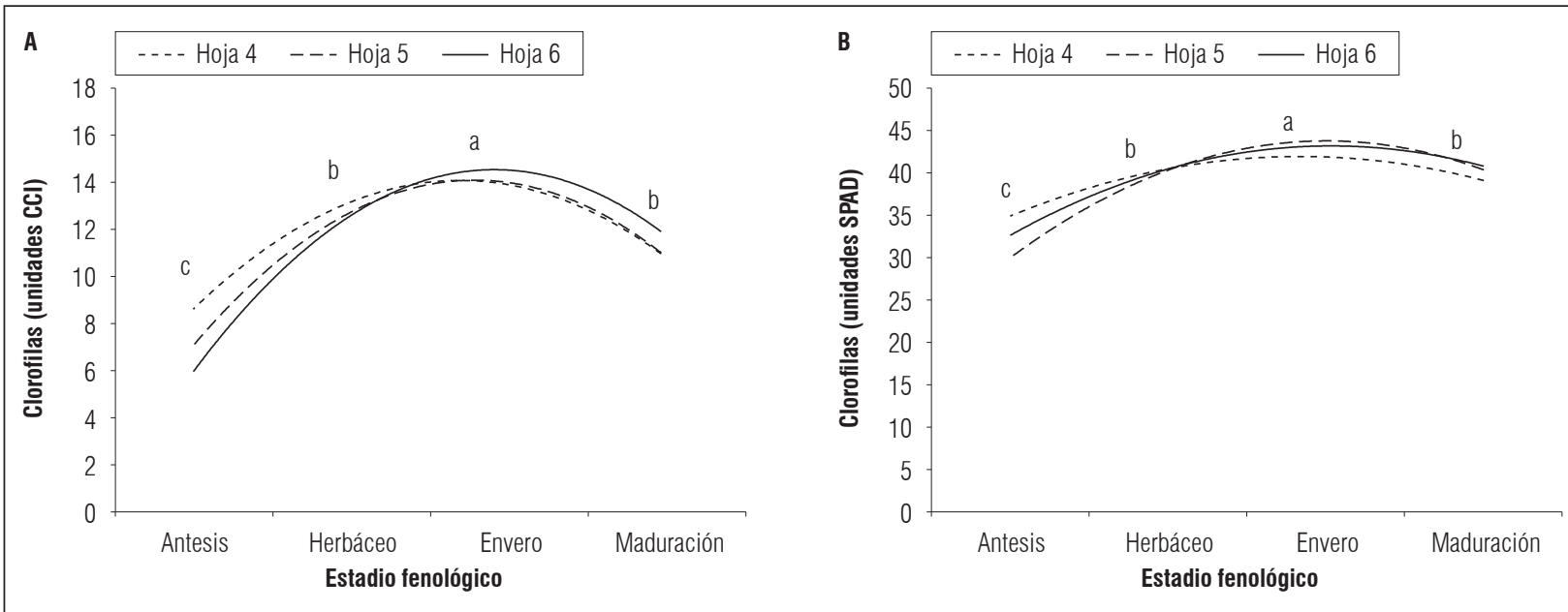

Figura 2. Contenido de clorofila: A. CCM-200 y B. SPAD 502, en diferentes estadios fenológicos Vitis vinifera L. cv. 'Riesling Becker' bajo condiciones de trópico alto. Promedios con letras distintas indican diferencia significativa según la prueba de Tukey $(P \leq 0,05)$.

Tabla 1. Ecuaciones de regresión para dos métodos de cuantificación no destructiva de clorofila en hojas de Vitis vinifera L. cv. 'Riesling Becker' bajo condiciones de trópico alto.

\begin{tabular}{|c|c|c|c|}
\hline Hoja & Ecuación método CCI & Ecuación método SPAD & $R^{2}$ \\
\hline 4 & $y=-1,8683 \times 2+10,124 x+0,3483$ & $y=-2,0917 \times 2+11,856 x+25,195$ & 0,9 \\
\hline 5 & $y=-2,1542 \times 2+12,08 x-2,8625$ & $y=-3,4458 \times 2+20,688 x+12,786$ & 0,9 \\
\hline 6 & $y=-2,2983 \times 2+13,472 x-5,2217$ & $y=-2,5608 \times 2+15,521 x+19,726$ & 0,9 \\
\hline
\end{tabular}

El contenido de clorofila se ajustó a polinomios de segundo grado para los dos métodos analizados con coeficientes de determinación $R^{2}$ superiores a 0,9 indicando que estos modelos son adecuados para explicar el comportamiento de las clorofilas al cuantificarlas mediante los dos métodos (Tab. 1).

En la investigación se encontró que a medida en que las hojas adquirían tonalidades de verde intenso, el valor de unidades SPAD y ICC aumentó (Fig. 2), es por ello que se hace importante tener en cuenta la planta y la hoja a evaluar a través de estos tipos de medidores con el fin de obtener una mayor confiabilidad en el resultado, la lectura de los medidores portátiles varía marcadamente entre hojas apicales de vid y hojas completamente expandidas (Fanizza et al., 1991).

Estos resultados concuerdan con lo encontrado por Allen et al. (2012), quienes indican que el contenido de clorofila, el área foliar y la absorción de la radiación aumentan en medida en que la hoja se hace fotosintéticamente madura. A su vez, Candolfi-Vasconcelos y Koblet (1991) indican que existe una tendencia en el comportamiento de la clorofila en plantas de vid, en el que se da un aumento gradual, alcanzando un máximo nivel en el estadio fenológico envero, y posteriormente en el estadio de maduración disminuye, posiblemente como respuesta de la planta a una adaptación por excesos de luz generado por una senescencia temprana de la hoja.

Por su parte, autores como Almanza-Merchán et al. (2012a) reportan como a medida que las hojas adquieren mayor desarrollo se convierten en las principales órganos de síntesis de fotosintatos, supliendo las demandas de los frutos, los cuales se convierten en demandantes de fotosintatos, durante los periodos herbáceo y envero; lo anterior concuerda con el contenido de clorofila de las hojas observado en el presente estudio.

Teniendo en cuenta los estudios realizados por Almanza-Merchán et al. (2012a), los cuales encontraron que en plantas de $V$. vinifera var. Riesling x Silvaner las hojas 4, 5 y 6 tienen un efecto directo sobre la calidad de los frutos gracias a la capacidad para retener 
el mayor contenido de solidos solubles totales y fotoasimilados traslocándolos hacia el racimo. En este contexto, se realizó un análisis en dichas hojas encontrando como se presentan los valores más altos en la hoja 4 durante el estadio fenológico antesis hasta llegar a envero, alcanzado valores de 12 unidades ICC, a partir de ahí y hasta llegar a la maduración los valores más altos se encontraban en la hoja 6 con valores de 16 unidades ICC, posiblemente causado por que las hojas 4 y 5 estaban entrando en un proceso de senescencia, donde finalmente para la etapa de cosecha la concentración de clorofila en las tres hojas tiende a estabilizarse, con valores cercanos a las 12 unidades ICC (Fig. 3).

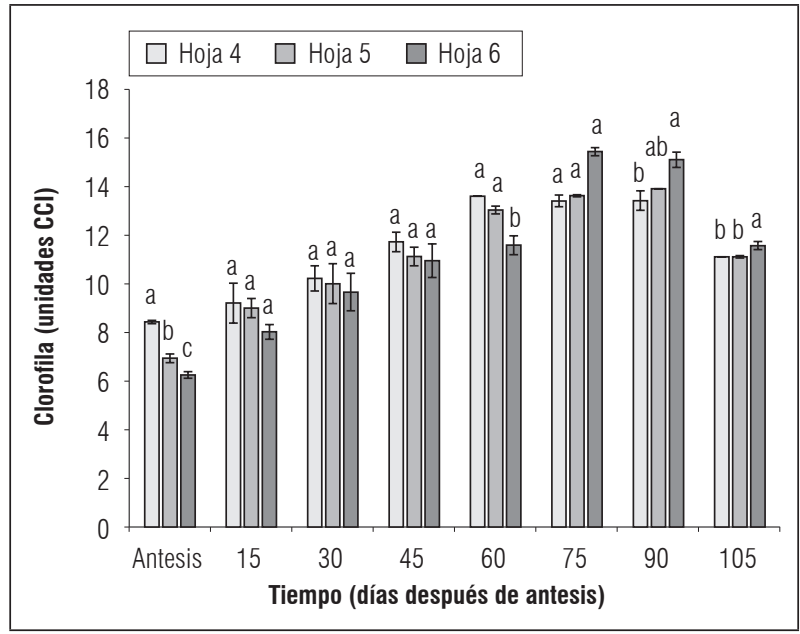

Figura 3. Contenido de clorofila cuantificado mediante medidor tipo CCM-200 ${ }^{\circledR}$ en hojas de Vitis vinifera L. Cv. 'Riesling Becker' bajo condiciones de trópico alto. Promedios con letras distintas indican diferencia significativa según la prueba de Tukey $(P \leq 0,05)$. Barras verticales indican error estándar $(n=3)$.

Teniendo en cuenta los resultados, se puede recomendar realizar evaluaciones de concentración de clorofila en hojas fotosintéticamente activas de plantas de vid presentes en el tercio medio de la planta, utilizando cualquiera de los dos equipos portátiles mencionados anteriormente. Dichas hojas se encuentran totalmente desarrolladas en los estadios fenológicos antesis, herbáceo y envero.

Resultados similares a los encontrados en el presente estudio fueron hallados por Bertamini y Nedunchezhian (2003), quienes indican cómo se presenta relación directa entre el área foliar, el contenido de clorofila y la absorción de la radiación, la cual va aumentando a medida en que la planta crece y se desarrolla.
Los mismos autores, también indican que en plantas de vid la concentración de clorofila tiene un aumento gradual alcanzando su máximo valor al llegar al estadio fenológico envero, con una disminución hasta llegar a la maduración de los frutos como consecuencia del proceso de senescencia del área foliar de la planta (Young et al., 2007; Callejas et al., 2013; Wang et al., 2016).

De acuerdo al contenido de clorofila encontrado en las hojas 4,5 y 6 cuantificado a través del medidor portátil de clorofila SPAD $502^{\circledR}$ (Fig. 4), se puede observar la misma tendencia encontrada con el equipo CCM-200 ${ }^{\circledR}$, teniendo la hoja 4 una tendencia creciente en la concentración del contenido de clorofila, donde presenta valores que van desde las 32 a 44 unidades SPAD hasta llegar al inicio del estadio fenológico envero o a los $45 \mathrm{~d}$ después de presentarse la antesis, donde posteriormente esta variable no presenta diferencias significativas en las tres hojas. Al iniciar el estadio fenológico de maduración (75 d), la concentración de clorofila se presenta de manera estable en estas hojas, las cuales presentan valores que van de los 40 a 45 unidades SPAD.

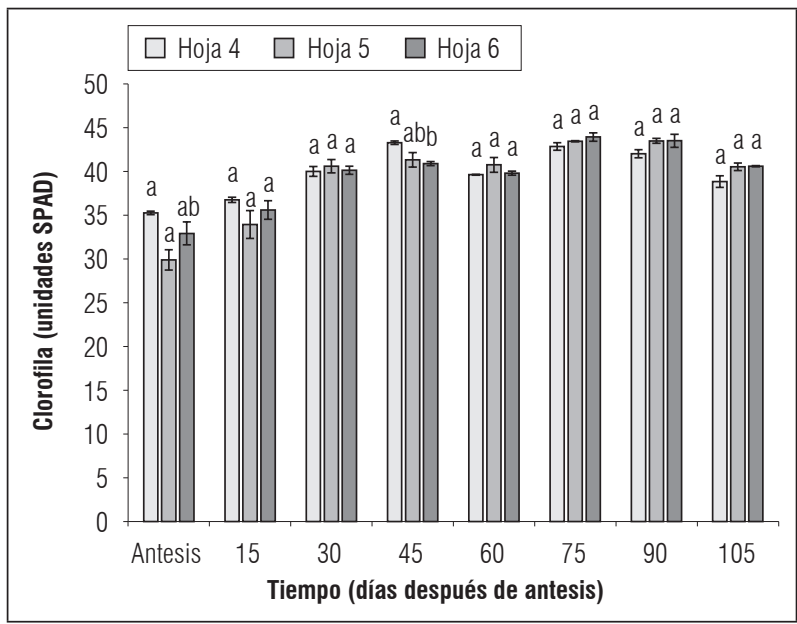

Figura 4. Contenido de clorofila cuantificado mediante medidor tipo SPAD $502{ }^{\circledR}$ en hojas de Vitis vinifera L. cv. 'Riesling Becker' bajo condiciones de trópico alto. Promedios con letras distintas indican diferencia significativa según la prueba de Tukey $(P \leq 0,05)$. Barras verticales indican error estándar $(n=3)$.

Los resultados encontrados en el presente estudio coinciden con los estudios realizados por Hunter y Visser (1989), quienes observaron que la mayor concentración de clorofila se encuentra en hojas cercanas al racimo durante los periodos de brotación y 
floración, así mismo en estas hojas se presentan las tasas más altas de eficiencia fotosintética. De acuerdo con esto, se puede afirmar la relación que existe entre el contenido de clorofila y la fotosíntesis, dado que la presencia de frutos estimula estos dos parámetros en vid (Iacono et al., 1995).

Las mediciones obtenidas con los dos equipos se ajustaron a un polinomio de segundo grado (Fig. 5), determinado por la ecuación para el índice de concentración de clorofila como ICC $=0,014 \mathrm{SPAD}^{2}-0,2396 \mathrm{~S}$ $\mathrm{PAD}+5,8021$. De acuerdo con modelo polinómico se obtuvo un valor de $R^{2}=0,9343(n=96 ; P \leq 0,0001)$, el cual indica que existe una alta correlación significativa y positiva entre los dos equipos portátiles de determinación de clorofila. Los resultados obtenidos en el presente trabajo coinciden con estudios realizados por Callejas et al. (2013), al comparar los mismos equipos en dos variedades de uva de mesa.

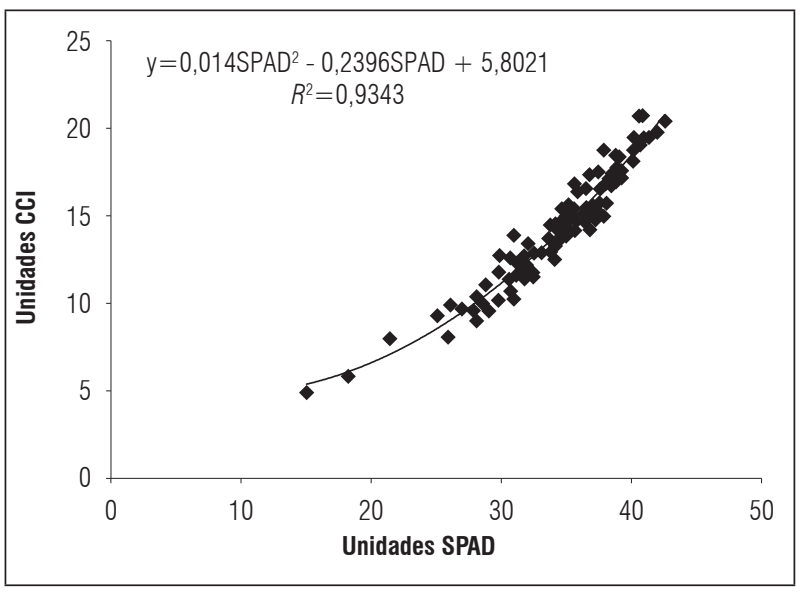

Figura 5. Correlación entre los métodos no destructivos CCM-200 ${ }^{\circledR}$ y SPAD $502{ }^{\circledR}$ para la cuantificación del contenido de clorofila en hojas de Vitis vinifera $L$. cv. 'Riesling Becker' bajo condiciones de trópico alto.

Las desigualdades encontradas en los dos equipos podrían estar relacionadas a las diferencias que poseen los equipos, principalmente a la variedad en las longitudes de onda emitidas y las áreas de evaluación de los equipos, donde el CCM-200 presenta un área de lectura de $0,71 \mathrm{~cm}^{2}$ con una longitud de onda que va desde 940 a $660 \mathrm{~nm}$ y $0,6 \mathrm{~cm}^{2}$ y 940 a $650 \mathrm{~nm}$ para el SPAD 502. A pesar de que este último posee un área de lectura de más o menos 10 veces menos, autores como Richardson et al. (2002) aseguran que no existe una mayor confiabilidad en el CCM-200 respecto del SPAD, lo cual fue demostrado en hojas de abedul, donde la mayor precisión la obtuvo el SPAD 502.

Con el fin de obtener una mayor confiablidad y un valor más distintivo, autores como Chang y Robinson (2003) sugieren corregir esta limitación a través de un muestreo más representativo por hoja, el cual posea más de cinco lecturas en cada una, con el fin de controlar la variabilidad dentro de la muestra y creando a su vez medidas más ajustadas.

\section{CONCLUSIONES}

Las evaluaciones realizadas con los medidores de clorofila SPAD $502^{\circledR}$ y CCM-200 ${ }^{\circledR}$ permiten obtener medidas rápidas y confiables, siempre y cuando que generen un número adecuado de mediciones, esto en relación a cada especie. En el presente estudio, cinco mediciones por hoja generaron un modelo ajustado. De igual manera, es importante la elección del estadio fenológico de la planta y del sector en el cual será realizada la medición, principalmente por la alta variabilidad en los contenidos de clorofila que se presentan en relación a las hojas y su filotaxia.

Conflicto de intereses: el manuscrito fue preparado y revisado con la participación de los autores, quienes declaran no tener algún conflicto de interés que coloquen en riesgo la validez de los resultados aquí presentados.

\section{REFERENCIAS BIBLIOGRÁFICAS}

Agronet. 2018. Análisis - Estadísticas, cultivo de Uva Colombia. En: Ministerio de Agricultura y Desarrollo Rural de Colombia, http://www.agronet.gov.co; consulta: junio de 2018.

Alizadeh, M., S.K. Singh, V.B. Patel, R.C. Bhattacharya y B.P. Yadav. 2010. In vitro responses of grape rootstocks to NaCl. Biol. Plant. 54, 381-385. Doi: 10.1007/ s10535-010-0069-0

Allen, F., T. Center y E. Mattison. 2012. In situ estimates of water hyacinth leaf tissue nitrogen using a SPAD502 chlorophyll meter. Aquat. Bot. 100, 72-75. Doi: 10.1016/j.aquabot.2012.03.005

Almanza-Merchán, P. 2011. Determinación del crecimiento y desarrollo del fruto de vid (Vitis vinifera L.) bajo condiciones de clima frío tropical. Tesis de doctorado. Facultad de Agronomía, Universidad Nacional de Colombia, Bogotá, Colombia.

Almanza-Merchán, P., S. González-Merchán y H.E. Balaguera-López. 2012a. La posición de la hoja y su efecto 
sobre la calidad y producción de frutos de vid (Vitis vinifera L.) var. Riesling $\times$ Silvaner. Rev. Colomb. Cienc. Hortic. 6(1), 9-18. Doi: 10.17584/rcch.2012v6i1.1283

Almanza-Merchan, P., P. Serrano y G. Fischer. 2012b. Manual de viticultura tropical colombiana. Universidad Pedagógica y Tecnológica de Colombia, Tunja, Colombia.

Arregui, L., M. Merina y A. Mingo. 2000. Aplicación del medidor portátil en los programas de fertilización nitrogenada en patata. pp. 157-170. En: Memorias Congreso Iberoamericano de Investigación y Desarrollo en Patata. Departamento de Producción Agraria, Universidad Pública de Navarra, Pamplona, España.

Bertamini, M. y N. Nedunchezhian. 2003. Photosynthetic functioning of individual grapevine leaves (Vitis vinifera L. cv. Pinot Noir) during ontogeny in the field. Vitis 42(1), 13-17.

Borhan, M.S., S. Panigrahi, M. Satter y H. Gu. 2017. Evaluation of computer imaging technique for predicting the SPAD readings in potato leaves. Inf. Process. Agr. 4(4), 275-282. Doi: 10.1016/j.inpa.2017.07.005

Callejas, R., E. Kania, A. Contreras, C. Peppi y L. Morales. 2013. Evaluación de un método no destructivo para estimar las concentraciones de clorofila en hojas de variedades de uva de mesa. Idesia 31(4), 19-26. Doi: 10.4067/S0718-34292013000400003

Candolfi-Vasconcelos, M. y W. Koblet. 1991. Influence of partial defoliation on gas exchange parameters and chlorophyll content on field grown grapevines mechanisms and limitations of the compensation capacity. Vitis 30, 129-141.

Cao, Q., Y. Miao, G. Feng, X. Gao, F. Li, B. Liu, S. Yue, S. Cheng, S. Ustin y R. Khosla. 2015. Active canopy sensing of winter wheat nitrogen status: an evaluation of two sensor systems. Comp. Elect. Agric. 112, 54-67. Doi: 10.1016/j.compag.2014.08.012

Cate, T. y T. Perkins. 2003. Chlorophyll content monitoring in sugar maple (Acer saccharum). Tree Physiol. 23, 1077-1079. Doi: 10.1093/treephys/23.15.1077

Chang, S. y D. Robinson. 2003. Nondestructive and rapid estimation of hardwood foliar nitrogen status using the SPAD-502 chlorophyll meter. Forest Ecol. Manage. 181(3), 331-338. Doi: 10.1016/S0378-1127(03)00004-5

Cho, Y.Y., S. Oh, M.M. Oh y J.E. Son. 2007. Estimation of individual leaf area, fresh weight, and dry weight of hydroponically grown cucumbers (Cucumis sativus L.) using leaf length, width, and SPAD value. Sci. Hortic. 111(4), 330-334. Doi: 10.1016/j.scienta.2006.12.028

Delegido, J., L. Alonso, G. González y J. Moreno. 2010. Estimating chlorophyll content of crops from hyperspectral data using a normalized area over reflectance curve (NAOC). Int. J. Appl. Earth Obs. Geoinf. 12(3), 165-174. Doi: 10.1016/j.jag.2010.02.003

Do Amarante, C., O. Zanuzo, A. Miqueloto, C. Steffens, J. Erhart y J. De Almeida. 2009. Quantificação da área e do teor de clorofilas emfolhas de plantas jovens de videira 'cabernet sauvignon' mediante métodos nãodestrutivos. Rev. Bras. Frutic. 31(3), 680-686. Doi: 10.1590/S0100-29452009000300009

Elarab, M., A. Ticlavilca., A. Torres-Rua., I. Maslova y M. McKee. 2015. Estimating chlorophyll with thermal and broadband multispectral high resolution imagery from an unmanned aerial system using relevance vector machines for precision agriculture. Int. J. Appl. Earth Obs. Geoinf. 43, 32-42. Doi: 10.1016/j. jag.2015.03.017

Fanizza, G., L. Ricciardi y C. Bagnulo. 1991. Leaf greenness measurements to evaluate water stressed genotypes in Vitis vinifera. Euphytica 55(1), 27-31. Doi: 10.1007/ BF00022556

Hawkins, T., E. Gardiner y G. Comer. 2009. Modeling the relationship between extractable chlorophyll and SPAD-502 readings for endangered plant species research. J. Nat. Conserv. 17(2), 123-127. Doi: 10.1016/j. jnc.2008.12.007

Hunter, J. y J. Visser. 1989. The effect of partial defoliation, leaf position and developmental stage of the vine on leaf chlorophyll concentration in relation to the photosynthetic activity and light intensity in the canopy of Vitis vinifera L. cv. Cabernet Sauvignon. S. Afr. J. Enol. Vitic. 10, 67-73. Doi: 10.21548/10-2-2289

Iacono, F., M. Bertamini, A. Scienza y B. Coombe. 1995. Differential effects of canopy manipulation and shading of Vitis vinifera L. cv. Cabernet Sauvignon. leaf gas exchange, photosynthetic electron transport rate and sugar accumulation in berries. Vitis 34(4), 201-206.

Kapotis, G., G. Zervoudakis, T. Veltsistas y G. Salahas. 2003. Comparaison of chlorophyll meter readings with leaf chlorophyll concentration in Amaranthus vlitus: correlation with physiological processes. Russ. J. Plant Physiol. 50(3), 395-397. Doi: 10.1023/A:1023886623645

Lorenz, D.H., K.W. Eichhorn, H. Blei-Holder, R. Klose, U. Meier y E. Weber. 1994. Phänologische entwicklungsstadien der weinrebe (Vitis vinifera L. ssp. vinifera). Vitic. Enol. Sci. 49, 66-70. Doi: 10.1111/j.1755-0238.1995. tb00085.x

Novoa, R. y A. Villagran. 2002. Evaluación de un instrumento medidor de clorofila en la determinación de niveles de nitrógeno foliar en maíz. Agric. Téc. 62(1), 165-171. Doi: 10.4067/S0365-28072002000100017

Ocon, P. 2008. Utilización del clorofilometro SPAD 502 para diagnosticar la deficiencia de nitrógeno en sorgo (Sorgum bicolor [L.] Moench) bajo distintas dosis de nitrógeno. Trabajo de grado. Facultad de Agronomía, Universidad Nacional Agraria, Managua.

Pinzón-Sandoval, E., I. Arias-Burgos y G. Cely-Reyes. 2017. Dinámica del crecimiento del fruto de vid (Vitis vinifera L.) cv 'Sauvignon' en trópico alto Colombiano. Cult. Cient. 15, 106-115. 
Quijano, M. 2004. Ecología de una conexión solar. De la adoración del sol al desarrollo vitivinícola regional. Cult. Cient. 2, 5-9.

Ramírez, V., A. Moreno y J. López. 2012. Evaluación temprana de la deficiencia del nitrógeno en café y aplicaciones. Avances Técnicos Cenicafe 420, 1-8.

Reyes, J., C. Correa y J. Zúñiga. 2017. Reliability of different color spaces to estimate nitrogen SPAD values in maize. Comp. Elect. Agric. 143, 14-22. Doi: 10.1016/j. compag.2017.09.032

Richardson, A., S. Duigan y G. Berlyn. 2002. Evaluation of noninvasive methods to estimate foliar chlorophyll content. New Phytol. 153, 185-194. Doi: 10.1046/j.0028-646X.2001.00289.x

Rincón, A. y G. Ligarreto. 2010. Relación entre nitrógeno foliar y el contenido de clorofila, en maíz asociado con pastos en el piedemonte llanero colombiano. Corpoica Cienc. Tecnol. Agropecu. 11(2), 122-128. Doi: 10.21930/rcta.vol11_num2_art:202

Rodríguez, M., G. Alcántar, A. Aguilar, J. Etchevers y J. Zantizó. 1998. Estimación de la concentración de nitrógeno y clorofila en tomate mediante un medidor portátil de clorofila. Terra Latinoam. 16(2), 135-141.

Romano, G., S. Zia, W. Spreer, C. Sanchez, J. Cairns, J. Araus y J. Müller. 2011. Use of thermography for high throughput phenotyping of tropical maize adaptation in water stress. Comp. Elect. Agric. 79, 67-74, Doi: 10.1016/j.compag.2011.08.011

Rosolem, C. y V. Van Mellis. 2010. Monitoring nitrogen nutrition in cotton. Rev. Bras. Ciênc. Solo 34(5), 1601 1607. Doi: 10.1590/S0100-06832010000500013
Sainz, H. y H. Echeverria. 1998. Relación entre las lecturas del medidor de clorofila (Minolta SPAD 502) en distintos estadios del ciclo del cultivo de maíz y el rendimiento en grano. Rev. Fac. Nac. Agron. Medellín 103(1), 37-44.

Salisbury, F. y C. Ross. 1992. Fisiología vegetal. Editorial Iberoamerica, México DF.

Senger, E., A. Peyrat, M. Martin y J. Montes. 2014. Genetic variation in leaf chlorophyll content of Jatropha curcas L. Ind. Crops Prod. 58, 204-211. Doi: 10.1016/j. indcrop.2014.04.003

Steele, M., A. Gitelson y D. Rundquist. 2007. A comparaison of two techniques for nondestructive measurement of chlorophyll content in grapevine leaves. Agron. J. 100(3), 779-782. Doi: 10.2134/agronj2007.0254N

Walteros, I.Y., D.C. Molano, P.J. Almanza-Merchán, M. Camacho y H.E. Balaguera-López. 2012. Efecto de la poda sobre la producción y calidad de frutos de Vitis vinifera L. var. Cabernet Sauvignon en Sutamarchán (Boyacá, Colombia). Rev. Colomb. Cienc. Hortic. 6(1), 19-30. Doi: 10.17584/rcch.2012v6i1.1279

Wang, H.-F., Z.-G. Huo, G.-S. Zhou, Q.-H. Liao, H.-K. Feng y L. Wu. 2016. Estimating leaf SPAD values of freeze-damaged winter wheat using continuous wavelet analysis. Plant Physiol. Biochem. 98, 39-45. Doi: 10.1016/j.plaphy.2015.10.032

Zulini, L., M. Rubinigg, R. Zorer y M. Bertamini. 2007. Effects of drought stress on chlorophyll fluorescente and photosynthetic pigment in grapevine leaves (Vitis vinifera cv. "White Riesling"). Acta Hortic. 754, 289294. Doi: 10.17660/ActaHortic.2007.754.37 